\title{
Long-term etanercept survival in patients with psoriatic arthritis: a multicenter retrospective analysis in daily clinical practice in Spain
}

\author{
Gustavo Deza ${ }^{1}$ - Jaime Notario ${ }^{2} \cdot$ Marta Ferran $^{1} \cdot$ Emma Beltrán $^{3} \cdot$ Miriam Almirall $^{3} \cdot$ Rebeca Alcalá ${ }^{1}$. \\ José Carlos Ruiz-Carrascosa ${ }^{4} \cdot$ Ricardo Sánchez $^{4} \cdot$ Silvia Pérez $^{5} \cdot$ María Luz García-Vivar $^{5} \cdot$ Eva Galíndez $^{5}$. \\ Maribel Mora ${ }^{6} \cdot$ Jesús Rodríguez $z^{6} \cdot$ Fernando Gallardo ${ }^{1}$ (I)
}

Received: 11 July 2018 / Accepted: 21 August 2018

(c) Springer-Verlag GmbH Germany, part of Springer Nature 2018

\begin{abstract}
Although several randomized clinical trials and observational studies have evaluated the effectiveness, safety and drug survival of etanercept (ETN) in the treatment of psoriatic arthritis (PsA), long-term data regarding these aspects are currently scarce. For this reason, we sought to investigate the long-term survival and safety of ETN in PsA patients in 4 tertiary care Spanish hospitals over a 13-year observation period (from 2004 to 2017). The records of 85 PsA patients were reviewed. ETN showed an excellent survival profile, with rates of treatment discontinuation at 1, 3, 5 and 10 years of 15, 37, 46 and $59 \%$, respectively. In our cohort, a trend toward longer drug survival in patients with shorter disease duration and those who were treated with ETN as their first biologic agent was observed. On the other hand, combination therapy with conventional disease-modifying antirheumatic drugs did not provide greater improvement on the long-term drug survival. Only $12 \%$ of the patients reported adverse events (AEs) during therapy, being most of them of mild to moderate intensity, and in only 7\% AEs led to drug discontinuation. To the best of our knowledge, the present study shows the largest follow-up period of ETN-treated population analyzed in a real-life setting, and these results demonstrate the positive safety profile and long-term effectiveness of this biologic agent in the management of PsA patients.
\end{abstract}

Keywords Arthritis $\cdot$ Etanercept $\cdot$ Psoriasis $\cdot$ Psoriatic $\cdot$ Survival $\cdot$ TNF- $\alpha$ blockers

\section{Introduction}

Psoriatic arthritis (PsA) is a chronic and heterogeneous disease characterized by inflammation of the joints, tendons and entheses [1, 2]. Its prevalence among Caucasian population is approximately $0.05-0.25 \%$, with an estimated incidence ranging from 3.6 to 7.2 per 100,000 person-years [1]. Due to pain, swelling and occasional joint deformity, PsA may lead to a significant functional impairment and profound impact on the patients' quality of life.

Conventional drug therapy of PsA has mainly consisted of non-steroidal anti-inflammatory drugs, regular physiotherapy, local injections of corticosteroids, and the conventional synthetic disease-modifying antirheumatic drugs

Gustavo Deza and Jaime Notario contributed equally to this work.

Fernando Gallardo

93702@parcdesalutmar.cat

Extended author information available on the last page of the article
(csDMARDs), such as methotrexate (MTX), sulfasalazine and leflunomide [2-4]. However, many patients remain uncontrolled despite these therapies [3]. The emergence of biological tumor necrosis factor- $\alpha$ (TNF- $\alpha$ ) inhibitors was a breakthrough in the management of PsA, since these agents have demonstrated their ability to induce a rapid and persistent clinical remission and improve many aspects of the disease [5-7]. As of yet, five TNF- $\alpha$ blockers are licensed for PsA management: etanercept (ETN), adalimumab (ADA), infliximab (IFX), golimumab and certolizumab, with the greater experience and data available on larger cohorts of patients for the first three drugs [8].

ETN is a recombinant, dimeric fusion protein consisting of two molecules of the soluble, extracellular ligand-binding portion of the p75 human TNF receptor linked to the Fc portion of human immunoglobulin $\mathrm{G}_{1}$ [9]. ETN blocks the interaction of TNF with receptors on the cell surface, prevents TNF-mediated inflammatory cellular responses and modulates the effects of other TNF-induced molecules [9]. Several randomized controlled trials (RCTs) have demonstrated the 
efficacy and safety of ETN in the treatment of PsA [7, 10]. During the past years, additional observational studies have also evaluated the effectiveness and drug survival (i.e. the length of time a patient continues to take a particular drug) of ETN in daily clinical practice [4, 8, 11-13]. However, long-term data regarding these aspects are currently scarce.

The primary aim of this study was to evaluate the longterm drug survival and safety of ETN in patients with PsA in a real-life setting over a 13-year observation period. The secondary objective was to identify clinical variables that could interfere on the long-term drug survival.

\section{Materials and methods}

\section{Study design and data collected}

This was a retrospective, multicenter, observational study that included adult patients who fulfilled the CASPAR criteria for PsA [14] consecutively treated with ETN at 4 tertiary care Spanish hospitals with PsA-specialized units. The study comprised the period from June 2004 to January 2017. Patients included failed to respond adequately to previous interventions and received ETN therapy according to the labelled indications and posology [15] for a minimum of 3 months after appropriate screening tests. The Clinical Research Ethics Committee of the centers involved granted ethical approval for the study (reference number: FGH-ETA-2014-01).

The following data were extracted from the hospital digital database: demographic data [e.g. age, gender and body mass index (BMI)], disease activity at the start of ETN therapy [measured by the disease activity score 28 using c-reactive protein (DAS28-CRP)] disease duration (defined as the time from PsA diagnosis to the start of ETN), pattern of articular involvement, presence of dactylitis and/or enthesitis, serum levels of c-reactive protein (CRP) and erythrocyte sedimentation rate (ESR) and detection of human leukocyte antigen B27 (HLA-B27), prior treatments, presence and severity (when appropriate) of cutaneous psoriasis ( $\mathrm{PsO}$ ), adverse events (AEs) reported during ETN therapy and number of patients who discontinued treatment. Reasons for ETN withdrawal were classified as: (i) lack of efficacy; (ii) AEs; and (iii) other reasons (moving to another reference center, lost to follow-up, etc.). Dermatological and/or rheumatological lack of efficacy was mainly determined by the clinical judgment of the dermatologists and rheumatologists of the specialized units for an inadequate disease control.

Duration of drug survival was calculated as the number of months during which individual patients continued their treatment with ETN. The start date was the date at which the first dose was administered, and the stop date was the date of the first missed dose. All observations were censored at
January 31, 2017. Temporary treatment interruptions (e.g. due to infections or surgery) of less than 3 months' duration were allowed.

\section{Statistical analysis}

Descriptive statistics were performed for each variable, using frequencies and percentages for categorical variables and median and range for quantitative variables. Categorical variables were compared between groups using Chisquared test, while quantitative variables were compared using Mann-Whitney $U$ test. Drug survival was estimated according to the Kaplan-Meier survival analysis and further analyzed with log-rank statistics. Cox proportional hazard models were used to investigate the effect of possible risk factors for treatment discontinuation. All analyses were made using SPSS version 22.0, and a $p$ value $<0.05$ was considered statistically significant.

\section{Results}

During the observation period, 85 PsA patients received treatment with ETN for a minimum of 3 months and, therefore, were included in the study. Clinical and demographic characteristics of the study population are summarized in Table 1. Thirty-nine patients (45.9\%) had typical features of $\mathrm{PsO}$ confirmed either by the rheumatologist or dermatologist (subjects showing cutaneous lesions without a clearly psoriasiform appearance were not labelled as PsO). Fifty-six patients $(65.9 \%)$ had not received previous treatment with other biologic agents, $51(60.0 \%)$ received ETN in monotherapy and 34 (40.0\%) with concomitant csDMARDs (MTX in 29 patients and leflunomide in 5). Most patients started ETN therapy at a dose of $25 \mathrm{mg}$ administered twice weekly or $50 \mathrm{mg}$ once weekly. However, in 12 patients (14.1\%) an initial induction (50 mg twice weekly) was used for up to 12 weeks due to concomitant moderate-to-severe PsO. On the other hand, 24 patients (28.2\%) optimized ETN therapy at a lower dosage rate (e.g. $25 \mathrm{mg}$ once weekly) during the follow-up period. Treatment with ETN was associated with a rapid improvement of the musculoskeletal symptoms, showing a significant reduction in the DAS28-CRP value at 6 months of treatment [median (range) value of DAS28-CRP at baseline and at 6 months: 3.0 (1.4-5.2) and 1.8 (0.5-4.1), respectively; $p<0.001]$.

Overall, 10 patients (11.8\%) experienced AEs during the follow-up period. The most common AEs were local reactions at the site of injection $(n=3)$. Other reported AEs included lower respiratory tract infections $(n=2)$, malignancies $(n=2)$, development of autoimmune disorders $(n=2)$ (one case of systemic sarcoidosis and one cutaneous lupus erythematosus) and uncomplicated headache $(n=1)$. 
Table 1 Clinical and demographic features of PsA patients included in the study

\begin{tabular}{|c|c|c|c|c|}
\hline Baseline characteristics & All patients $(n=85)$ & $\begin{array}{l}\text { Patients who discontin- } \\
\text { ued ETN }(n=44)\end{array}$ & $\begin{array}{l}\text { Patients who maintained } \\
\text { ETN }(n=41)\end{array}$ & $p$ value \\
\hline Female sex, $n(\%)$ & $33(38.8)$ & $19(43.2)$ & $14(34.1)$ & 0.393 \\
\hline Age (years), median (range) & $58(32-89)$ & $57(34-84)$ & $58.5(32-89)$ & 0.247 \\
\hline Obesity [BMI $\left.\left(\mathrm{kg} / \mathrm{m}^{2}\right) \geq 30\right], n(\%)$ & $34(40.0)$ & $19(43.2)$ & $15(36.6)$ & 0.535 \\
\hline Concomitant PsO, $n(\%)$ & $39(45.9)$ & $17(38.6)$ & $22(53.7)$ & 0.165 \\
\hline Plaque $\mathrm{PsO}, n(\%)$ & $29(74.4)$ & $11(64.7)$ & $18(81.8)$ & \\
\hline Inverse $\mathrm{PsO}, n(\%)$ & $3(7.7)$ & $1(5.9)$ & $2(9.1)$ & \\
\hline Other variants of $\mathrm{PsO}, n(\%)$ & $7(17.9)$ & $5(29.4)$ & $2(9.1)$ & \\
\hline PASI (if concomitant PsO), median (range) & $13(2-30)$ & $15(2-30)$ & $12.8(2-24)$ & 0.557 \\
\hline \multicolumn{5}{|l|}{ Comorbidities } \\
\hline Diabetes mellitus, $n(\%)$ & $17(20.0)$ & $10(22.7)$ & $7(17.1)$ & 0.515 \\
\hline Hyperlipidemia, $n(\%)$ & $28(32.9)$ & $14(31.8)$ & $14(34.1)$ & 0.819 \\
\hline Arterial hypertension, $n(\%)$ & $36(42.4)$ & $18(40.9)$ & $18(43.9)$ & 0.780 \\
\hline Peripheral arthritis, $n(\%)$ & $74(87.1)$ & $40(90.1)$ & $34(82.9)$ & 0.273 \\
\hline Polyarticular pattern, $n(\%)$ & $66(77.6)$ & $31(70.5)$ & $35(85.4)$ & 0.099 \\
\hline Axial involvement, $n(\%)$ & $33(38.8)$ & $13(29.5)$ & $20(48.8)$ & 0.069 \\
\hline Dactylitis and/or enthesitis of heel, $n(\%)$ & $23(27.1)$ & $15(34.1)$ & $8(19.5)$ & 0.131 \\
\hline PsA activity (DAS28-CRP), median (range) & $3.0(1.4-5.2)$ & $3.1(1.4-5.2)$ & $2.7(1.7-5.0)$ & 0.509 \\
\hline Disease duration (months), median (range) & $72(12-468)$ & $96(12-468)$ & $60(12-360)$ & 0.055 \\
\hline Positive HLA-B27, $n(\%)$ & $5(5.9)$ & $4(9.1)$ & $1(2.4)$ & 0.193 \\
\hline ESR serum levels $(\mathrm{mm} / \mathrm{h})$, median (range) & $15(2-89)$ & $19(2-89)$ & $11(3-56)$ & 0.069 \\
\hline CRP serum levels (mg/dL), median (range) & $1.9(0.1-35.4)$ & $3.6(0.1-35.4)$ & $1.3(0.1-30.0)$ & 0.227 \\
\hline Previous treatment with biologic agents, $n(\%)$ & $29(34.1)$ & $18(40.9)$ & $11(26.8)$ & 0.171 \\
\hline Co-medication with csDMARDs, $n(\%)$ & $34(40.0)$ & $19(43.2)$ & $15(36.6)$ & 0.535 \\
\hline Adverse events, $n(\%)$ & $10(11.8)$ & $8(18.2)$ & $2(4.9)$ & 0.057 \\
\hline Infection, $n(\%)$ & $2(20.0)$ & $0(0)$ & $2(100)$ & \\
\hline Injection site reaction, $n(\%)$ & $3(30.0)$ & $3(37.5)$ & $0(0)$ & \\
\hline Headache, $n(\%)$ & $1(10.0)$ & $1(12.5)$ & $0(0)$ & \\
\hline Malignancy, $n(\%)$ & $2(20.0)$ & $2(25.0)$ & $0(0)$ & \\
\hline Autoimmune disorder, $n(\%)$ & $2(20.0)$ & $2(25.0)$ & $0(0)$ & \\
\hline
\end{tabular}

ETN etanercept, BMI body mass index, PsO cutaneous psoriasis, $P A S I$ psoriasis area and severity index, PsA psoriatic arthritis, DAS28-CRP disease activity score 28 using c-reactive protein, $H L A$ human leukocyte antigen, $E S R$ erythrocyte sedimentation rate, $C R P$ c-reactive protein, csDMARDs conventional synthetic disease-modifying antirheumatic drugs

Regarding the status of ETN use throughout the follow-up period, 41 patients (48.2\%) maintained ETN therapy with a median (range) time of 98 months (28-154 months), while $44(51.8 \%)$ discontinued treatment with a median (range) time of 24 months (3-109 months). No significant differences regarding clinical and demographic features were found between these two groups (Table 1). Reasons for ETN discontinuation were lack of efficacy in $84.1 \%$ of the cases ( $n=37$; in which 31 and 6 cases were due to rheumatological and dermatological lack of efficacy, respectively), AEs in $13.6 \%$ [injection site reactions $(n=2)$, malignancies $(n=2)$, autoimmune disorder $(n=1)$ and headache $(n=1)]$ and lost to follow-up in $2.3 \%(n=1)$.

Drug survival rates estimated by the Kaplan-Meier analysis are shown in Fig. 1. In our study population, rates of treatment discontinuation found at 1,2 and 3 years were
15, 27 and 37\%, respectively. This positive survival profile was maintained at long-term follow-up, with discontinuation rates at 5, 7.5 and 10 years of 46,55 and 59\%, respectively. Regarding the potential predictors of ETN withdrawal (Table 2), the previous use of biologic therapies was the unique variable related to treatment discontinuation $(p=0.028)$. A non-significant trend towards longer drug survival in patients with shorter disease duration was also observed $(p=0.073)$.

\section{Discussion}

Although RCTs are considered the gold standard for assessing efficacy and safety of a specific therapeutic intervention, their results are often limited due to their brief follow-up 
Fig. 1 Drug survival of ETN treatment in PsA shown as the fraction (between 1 and 0 ) of patients remaining on therapy during the observation period: $\mathbf{a}$ in all PsA patients included in the study; $\mathbf{b}$ in patients with and without prior exposure to biologic agents $(p=0.024)$, and $\mathbf{c}$ in patients receiving treatment with ETN in monotherapy or in combination with csDMARDs $(p=0.334)$

time and stringent inclusion criteria. Therefore, findings based on the real-world evidence including more diverse populations and longer follow-up periods are of utmost importance. In this regard, during the past years, several observational studies have evaluated the drug survival of TNF- $\alpha$ blockers in the treatment of PsA in a real-life setting. Drug survival is considered a measure of therapeutic success as it is largely conditioned by drug efficacy and tolerance. Such reports, in overall, reflect the good survival profile of these agents in PsA patients, with rates of treatment discontinuation ranging from 12 to $30 \%$ at 12 months and from 19 to $43 \%$ at 24 months $[4,8,12,13,16-18]$. Similar adherence has been shown for ETN therapy in PsA, with discontinuation rates between $14-19,21-27$ and $33-35 \%$ at 12,24 and 36 months, respectively $[12,13]$. In this particular clinical setting, ETN seems to exhibit a better drug survival than other TNF- $\alpha$ blockers $[8,11,19]$. Although these findings support the effectiveness and safety of ETN in PsA in routine clinical practice, long-term data, which would provide very useful information for the management of PsA, are currently scarce. Indeed, to our knowledge, the present study shows the largest follow-up period of a ETN-treated population analyzed in a real-life setting, with a 13-year observation period and a median drug survival of more than 50 months. Based on our results, ETN shows persistent long-term effectiveness and limited toxicity. With similar discontinuation rates at 1,2 and 3 years of treatment compared to previous reports, this excellent survival profile of ETN was also maintained at long-term of follow-up. ETN also showed an excellent long-term safety and tolerability. Thus, in our cohort, only $12 \%$ of the patients reported AEs during therapy, being most of them of mild to moderate intensity, and in only $7 \%$ AEs led to drug discontinuation.

In patients with chronic inflammatory diseases, TNF- $\alpha$ blockers are often administered in combination with csDMARDs, particularly MTX, to enhance clinical outcomes, reduce risk of immunogenicity and improve drug survival $[20,21]$. Proposed mechanisms of this phenomenon are the synergistic pharmacological effects and the reduction of the effect of potential antibodies against the biologic agent [21]. Co-medication with MTX or other csDMARDs is also a common practice among PsA patients during treatment with TNF- $\alpha$ blockers [21]. Although it has been shown that this combination therapy does not provide greater improvement of PsA clinical symptoms compared to anti-TNF monotherapy, a more prolonged drug survival may be expected in
A

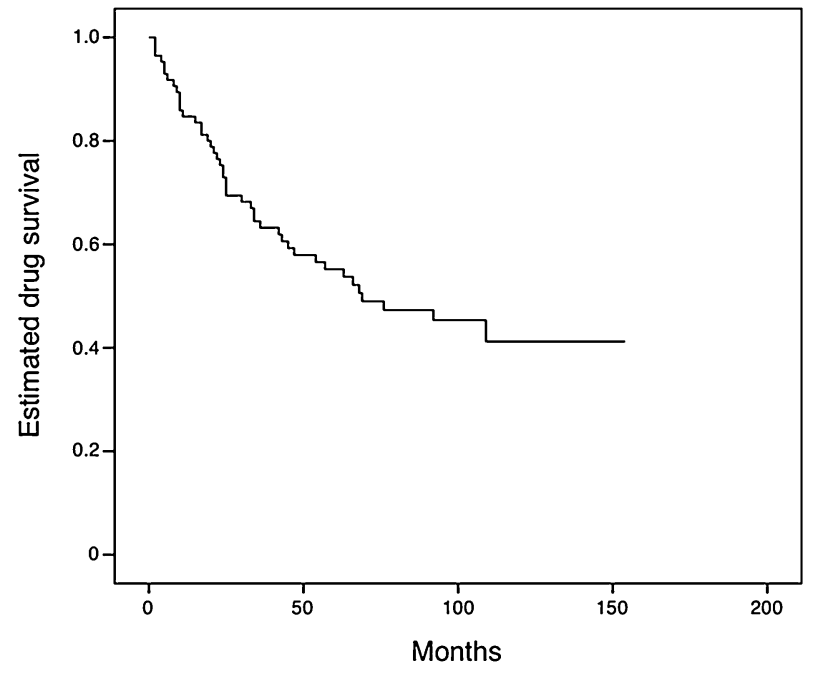

B
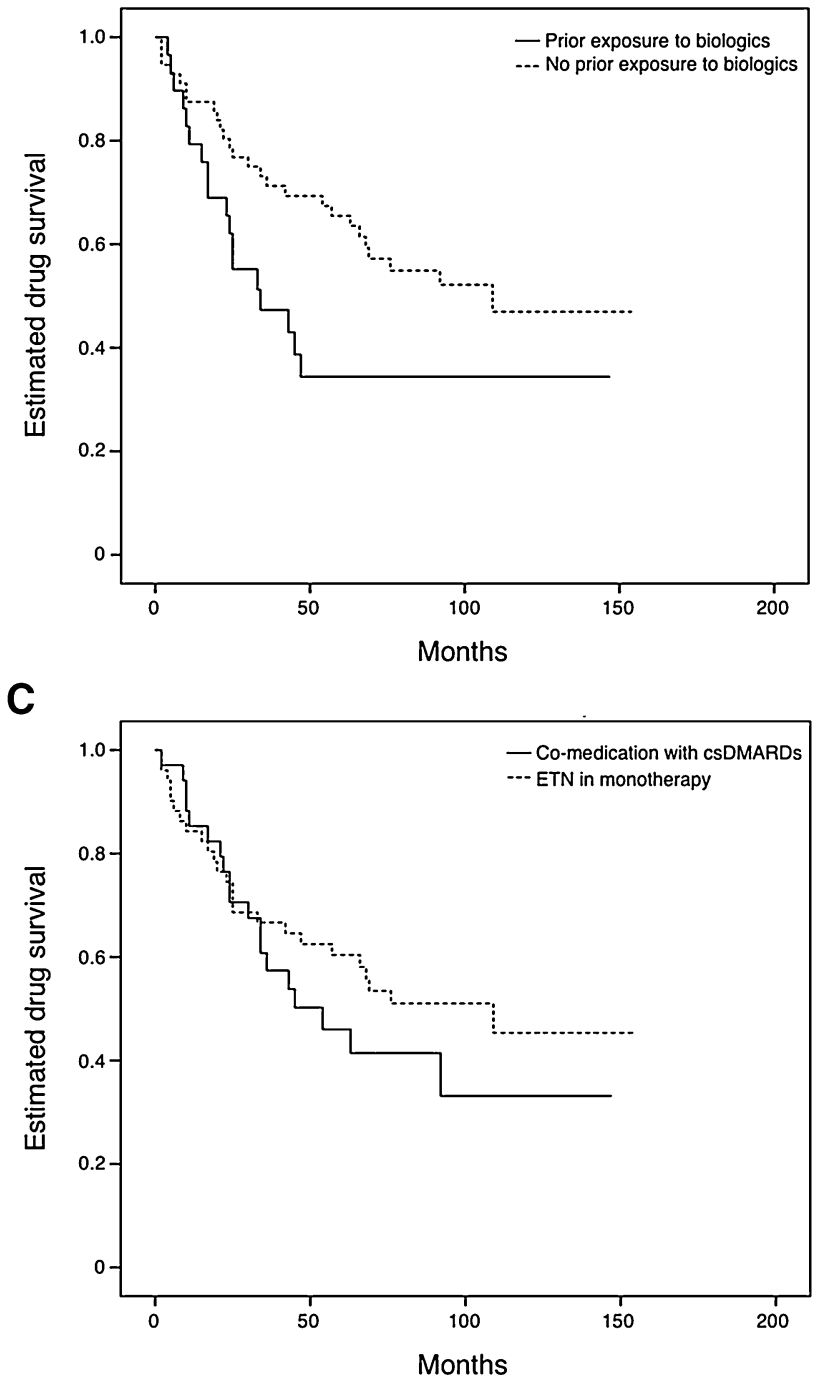
Table 2 Predictors of treatment discontinuation in PsA patients

\begin{tabular}{lll}
\hline Baseline characteristics & HR $(95 \% \mathrm{CI})$ & $p$ value \\
\hline Sex: female vs. male & $1.13(0.62-2.05)$ & 0.695 \\
Age (years): $\geq 65$ vs. $<65$ & $1.11(0.55-2.22)$ & 0.773 \\
BMI $\left(\mathrm{kg} / \mathrm{m}^{2}\right): \geq 30$ vs. $<30$ & $1.00(0.53-1.90)$ & 0.999 \\
Concomitant PsO: yes vs. no & $0.71(0.39-1.31)$ & 0.277 \\
Severe PsO: PASI $\geq 10$ vs $<10$ & $0.66(0.18-2.46)$ & 0.533 \\
PsA activity: DAS28-CRP $\geq 3$ vs $<3$ & $1.07(0.49-2.36)$ & 0.861 \\
Disease duration (months): $\geq 60$ vs. $<60$ & $1.92(0.94-3.92)$ & 0.073 \\
ESR serum levels (mm/h): $\geq 12$ vs. $<12$ & $1.34(0.64-2.80)$ & 0.437 \\
CRP serum levels (mg/dL): $\geq 1$ vs. $<1$ & $1.55(0.74-3.25)$ & 0.242 \\
Co-medication with csDMARDs: yes & $1.34(0.74-2.46)$ & 0.338 \\
$\quad$ vs. no & & \\
Prior biologic treatment: no vs. yes & $0.50(0.27-0.93)$ & $0.028 *$ \\
\hline
\end{tabular}

$H R$ hazard ratio, $C I$ confidence interval, $B M I$ body mass index, $P s O$ cutaneous psoriasis, $P A S I$ psoriasis area and severity index, $P S A$ psoriatic arthritis, $D A S 28-C R P$ disease activity score 28 using c-reactive protein, $E S R$ erythrocyte sedimentation rate, $C R P$ c-reactive protein, cSDMARDs conventional synthetic disease-modifying antirheumatic drugs

*Statistically significant

patients receiving co-medication $[4,17,19,21]$. However, for ETN treatment specifically, some previous reports failed to demonstrate this beneficial effect in terms of drug survival [20-22], which is consistent with our results. A possible explanation for this finding is that the formation of antidrug antibodies, which is supposedly one of the main targets of csDMARDs co-medication, is rarely detected during ETN therapy compared to others TNF- $\alpha$ blockers [23]. Given the presumed absence of beneficial effects of the combination therapy and the risk of AEs associated with treatment with MTX or other csDMARDs, this observation deserves further investigations as it might have important implications in clinical practice.

Some other clinical variables have been proposed as potential predictors of drug discontinuation in PsA patients treated with TNF- $\alpha$ blockers. For example, high CRP serum levels at baseline have been associated with longer drug survival [4, 19], while female gender, older age, or higher baseline disease activity has been linked to a premature drug discontinuation $[4,8,12,17,24]$. Although such observations could not be corroborated in our study, we found that disease duration and the previous use of biologic therapies may also have influence on the long-term survival of ETN in PsA patients. Thus, a trend toward longer drug survival in patients with shorter disease duration and those who were treated with ETN as their first biologic agent was observed in our cohort. Previous data have also suggested that patients receiving their second TNF- $\alpha$ blocker may have significantly poorer clinical responses and lower drug survival compared to patients receiving TNF- $\alpha$ blockers as their first biologic agent [25]. These findings, taken together, do not imply that ETN should not be attempted in those who fail a previous biologic treatment, but should be taken into account for a proper patient's counseling and planning of PsA management.

Limitations of this observational study are mainly related to its retrospective nature, which may lead to missing or incomplete data. The limited number of patients included and the non-use of objective scales to evaluate the lack of therapeutic efficacy constitute additional limitations. On the other hand, the strengths of our study are based on data collection from different centers in a real-life clinical setting and the long follow-up period.

In summary, the results from our 13-year experience with PsA patients treated with ETN confirm the positive safety profile and long-term effectiveness previously shown in RCTs and observational studies. In our cohort, a trend toward longer drug survival in patients with shorter disease duration and those who were treated with ETN as their first biologic was observed. On the other hand, concomitant csDMARDs might not provide greater improvement on the long-term ETN survival in PsA patients. This finding may be particularly important in patients with intolerance or contraindications to MTX or other csDMARDs.

Author contributions GD registration of patients and drafted the manuscript. JN registration and management of patients and drafted the manuscript. MF registration and management of patients and manuscript revision. EB registration and management of patients and manuscript revision. MA registration and management of patients and manuscript revision. RA registration of patients, statistical analyses and manuscript revision. JCRC registration and management of patients and manuscript revision. RS Registration and management of patients and manuscript revision. SP registration and management of patients and manuscript revision. MLG-V registration and management of patients and manuscript revision. EG registration and management of patients and manuscript revision. MM registration and management of patients and manuscript revision. JR registration and management of patients and manuscript revision. FG management of patients, study's design and manuscript supervision.

Funding This study was supported by an Investigator Initiated Research (IIR) from Pfizer (etanercept manufacturer), providing economic support (Grant ID: WI195272) to carry out the study. Pfizer did not have access to the patients' data or data analysis. The analysis of the data and the writing of the manuscript were done entirely by the authors.

\section{Compliance with ethical standards}

Conflict of interest No conflicts of interest to declare.

\section{References}

1. Ogdie A, Weiss P (2015) The epidemiology of psoriatic arthritis. Rheum Dis Clin N Am 41:545-568 
2. Gossec L, Smolen JS, Ramiro S et al (2016) European League Against Rheumatism (EULAR) recommendations for the management of psoriatic arthritis with pharmacological therapies: 2015 update. Ann Rheum Dis 75:499-510

3. Ritchlin CT, Kavanaugh A, Gladman DD et al (2009) Treatment recommendations for psoriatic arthritis. Ann Rheum Dis 68:1387-1394

4. Glintborg B, Østergaard M, Dreyer L et al (2011) Treatment response, drug survival, and predictors thereof in 764 patients with psoriatic arthritis treated with anti-tumor necrosis factor $\alpha$ therapy: results from the nationwide Danish DANBIO registry. Arthritis Rheum 63:382-390

5. Antoni C, Krueger GG, de Vlam K et al (2005) Infliximab improves signs and symptoms of psoriatic arthritis: results of the IMPACT 2 trial. Ann Rheum Dis 64:1150-1157

6. Mease PJ, Gladman DD, Ritchlin CT et al (2005) Adalimumab for the treatment of patients with moderately to severely active psoriatic arthritis: results of a double-blind, randomized, placebocontrolled trial. Arthritis Rheum 52:3279-3289

7. Mease PJ, Kivitz AJ, Burch FX et al (2004) Etanercept treatment of psoriatic arthritis: safety, efficacy, and effect on disease progression. Arthritis Rheum 50:2264-2272

8. Iannone F, Lopriore S, Bucci R et al (2015) Two-year survival rates of anti-TNF- $\alpha$ therapy in psoriatic arthritis (PsA) patients with either polyarticular or oligoarticular PsA. Scand J Rheumatol 44:192-199

9. Scott LJ (2014) Etanercept: a review of its use in autoimmune inflammatory diseases. Drugs 74:1379-1410

10. Mease PJ, Goffe BS, Metz J et al (2000) Etanercept in the treatment of psoriatic arthritis and psoriasis: a randomised trial. Lancet 356:385-390

11. López-González R, León L, Loza E et al (2015) Adherence to biologic therapies and associated factors in rheumatoid arthritis, spondyloarthritis and psoriatic arthritis: a systematic literature review. Clin Exp Rheumatol 33:559-569

12. Saad AA, Ashcroft DM, Watson KD et al (2009) Persistence with anti-tumour necrosis factor therapies in patients with psoriatic arthritis: observational study from the British Society of Rheumatology Biologics Register. Arthritis Res Ther 11:R52

13. Aaltonen K, Heinonen A, Joensuu J et al (2017) Effectiveness and drug survival of TNF-inhibitors in the treatment of psoriatic arthritis: a prospective cohort study. Semin Arthritis Rheum 46:732-739
14. Taylor W, Gladman D, Helliwell P et al (2006) Classification criteria for psoriatic arthritis: development of new criteria from a large international study. Arthritis Rheum 54:2665-2673

15. Spadaro A, Lubrano E, Ferrara N et al (2012) Etanercept in psoriatic arthritis. J Rheumatol 89:74-76

16. Virkki LM, Sumathikutty BC, Aarnio M et al (2010) Biological therapy for psoriatic arthritis in clinical practice: outcomes up to 2 years. J Rheumatol 37:2362-2368

17. Heiberg MS, Koldingsnes W, Mikkelsen K et al (2008) The comparative one-year performance of anti-tumor necrosis factor alpha drugs in patients with rheumatoid arthritis, psoriatic arthritis, and ankylosing spondylitis: results from a longitudinal, observational, multicenter study. Arthritis Rheum 59:234-240

18. Carmona L, Gómez-Reino JJ, BIOBADASER Group (2006) Survival of TNF antagonists in spondylarthritis is better than in rheumatoid arthritis. Data from the Spanish registry BIOBADASER. Arthritis Res Ther 8:R72

19. Kristensen LE, Gülfe A, Saxne T et al (2008) Efficacy and tolerability of anti-tumour necrosis factor therapy in psoriatic arthritis patients: results from the South Swedish Arthritis Treatment Group register. Ann Rheum Dis 67:364-369

20. Combe B, Behrens F, McHugh N et al (2016) Comparison of etanercept monotherapy and combination therapy with methotrexate in psoriatic arthritis: results from 2 clinical trials. J Rheumatol 43:1063-1067

21. Fagerli KM, Lie E, van der Heijde D et al (2014) The role of methotrexate co-medication in TNF-inhibitor treatment in patients with psoriatic arthritis: results from 440 patients included in the NOR-DMARD study. Ann Rheum Dis 73:132-137

22. de Vlam K, Boone C, The Prove Study Group A (2015) Treatment adherence, efficacy, and safety of etanercept in patients with active psoriatic arthritis and peripheral involvement in Belgium for 66 months (PROVE study). Clin Exp Rheumatol 33:624-631

23. Zisapel M, Zisman D, Madar-Balakirski N et al (2015) Prevalence of TNF- $\alpha$ blocker immunogenicity in psoriatic arthritis. J Rheumatol 42:73-78

24. Gomez-Reino JJ, Carmona L, BIOBADASER Group (2006) Switching TNF antagonists in patients with chronic arthritis: an observational study of 488 patients over a four-year period. Arthritis Res Ther 8:R29

25. Fagerli KM, Lie E, van der Heijde D et al (2013) Switching between TNF inhibitors in psoriatic arthritis: data from the NORDMARD study. Ann Rheum Dis 72:1840-1844

\section{Affiliations}

\section{Gustavo Deza ${ }^{1}$ (1) - Jaime Notario ${ }^{2} \cdot$ Marta Ferran $^{1} \cdot$ Emma Beltrán $^{3} \cdot$ Miriam Almirall $^{3} \cdot$ Rebeca Alcalá $^{1}$. José Carlos Ruiz-Carrascosa ${ }^{4} \cdot$ Ricardo Sánchez $^{4}$ - Silvia Pérez ${ }^{5}$ - María Luz García-Vivar ${ }^{5}$. Eva Galíndez ${ }^{5}$. Maribel Mora ${ }^{6} \cdot$ Jesús Rodríguez $^{6} \cdot$ Fernando Gallardo $^{1}(\mathbb{D}$}

Gustavo Deza

gustavo.deza@gmail.com

Jaime Notario

jnotario@bellvitgehospital.cat

Marta Ferran

MFerran@parcdesalutmar.cat

Emma Beltrán

EBeltran@parcdesalutmar.cat

Miriam Almirall

miriamalmirall1974@gmail.com
Rebeca Alcalá

rebeca.alcala@gmail.com

José Carlos Ruiz-Carrascosa

ruizcarrascosa@movistar.es

Ricardo Sánchez

rsparera@gmail.com

Silvia Pérez

Silvia.perezbarrio@ osakidetza.net

María Luz García-Vivar

marialuz.garciavivar@osakidetza.net 
Eva Galíndez

evagalindez@gmail.com

Maribel Mora

mmora@bellvitgehospital.cat

Jesús Rodríguez

jesus.rodriguez@bellvitgehospital.cat

1 Department of Dermatology, Hospital del Mar-Institut Mar d'Investigacions Mèdiques, Passeig Marítim, 25-29, 08003 Barcelona, Spain

2 Department of Dermatology, Hospital de Bellvitge, Hospitalet de Llobregat, Spain
3 Department of Reumatology, Hospital del Mar, Barcelona, Spain

4 Department of Reumatology, Hospital San Cecilio, Granada, Spain

5 Department of Reumatology, Hospital de Basurto, Bilbao, Spain

6 Department of Reumatology, Hospital de Bellvitge, Hospitalet de Llobregat, Spain 